\title{
ENHANCEMENT FACTORS FOR ELECTRONS IN SOLIDS FOR SOME SIMPLE CASES
}

\author{
H. Stachowiak, E. Boroński and G. Banach \\ W. Trzebiatowski Institute for Low Temperature and Structure Research \\ Polish Academy of Sciences, P.O. Box 1410, 50-950 Wrocław 2, Poland
}

\begin{abstract}
The approach to $\mathrm{e}^{+}-\mathrm{e}^{-}$interaction in jellium proposed by Gondzik and Stachowiak proved to be simple numerically and leading to reasonable results. This is why it seemed to be particularly suited for generalizations to real solids, a problem that in spite of more than thirty years of research in this direction did not get yet a satisfactory solution. An obstacle up to now was the necessity to solve integro-differential equations in two dimensions. After overcoming this difficulty we were able to compute $\mathrm{e}^{+}-\mathrm{e}^{-}$enhancement for core and valence electrons in lithium and for core electrons in other two-electron core elements. Properties of the electronic cloud screening the positron are investigated for different coordinates of this particle. The results are compared to such simplifying guesses concerning this problem as the local density, the generalized gradient and the weighted density approximations.
\end{abstract}

PACS numbers: $71.60 .+\mathrm{z}, 78.70 . \mathrm{Bj}$

\section{Introduction}

Many-body approaches to the problem of electron-positron interaction (EPI) in metal lattices did not lead to conclusive results $[1,2]$. This is why approaches to this effect were developed which benefit of the results obtained for EPI in an electron gas. Let us mention here the local density approximation (LDA) [3-6], the generalized gradient approximation (GGA) [7], and the weighted density approximation (WDA) [8] (for comparison of the above approaches with experiment cf. [9]).

The approach of Gondzik and Stachowiak to EPI in jellium [10] which bases on the results of the theory of liquids [11] is relatively simple and leads to reasonable results for the annihilation rate for all electron densities. This is why we applied it to study EPI in light elements. The problem is sufficiently complicated for avoiding the additional obstacle connected with the metal lattice that disturbs the spherical symmetry of the system. Therefore let us demonstrate this approach using the example of an atom of lithium embedded in an electron gas. 
We describe the electronic structure of this system using two functions $\psi_{1}(r)$ and $\psi_{2}(r) . \psi_{1}(r)$ is the wave function of core electrons and $\psi_{2}(r)$ is the density amplitude of valence electrons. $\psi_{2}^{2}$ determines the density of conduction electrons in the model [12]. These two functions obey the appropriate Kohn-Sham-like equations (in atomic units that will be used throughout this work)

$$
\left[-\frac{1}{2} \nabla^{2}+V(\boldsymbol{r})\right] \psi_{1}(\boldsymbol{r})=E_{1} \psi_{1}(\boldsymbol{r}), \quad\left[-\frac{1}{2} \nabla^{2}+V(\boldsymbol{r})\right] \psi_{2}(\boldsymbol{r})=0
$$

where

$$
\begin{aligned}
& V(\boldsymbol{r})=-\frac{Z}{r}+2 \int \mathrm{d} \boldsymbol{r}^{\prime} \frac{\psi_{1}^{2}\left(\boldsymbol{r}^{\prime}\right)}{\left|\boldsymbol{r}-\boldsymbol{r}^{\prime}\right|}+\int \mathrm{d} \boldsymbol{r}^{\prime} \frac{\psi_{2}^{2}\left(\boldsymbol{r}_{p}, \boldsymbol{r}^{\prime}\right)-d\left(\boldsymbol{r}^{\prime}\right)}{\left|\boldsymbol{r}-\boldsymbol{r}^{\prime}\right|} \\
& +V_{\mathrm{HL}}\left[2 \psi_{1}^{2}(\boldsymbol{r})+\psi_{2}^{2}(\boldsymbol{r})\right]-V_{\mathrm{HL}}\left(\rho_{0}\right) .
\end{aligned}
$$

$Z$ is the charge of the nucleus, $d(r)$ is the distribution of the positive charge in the electron gas. It is equal to

$$
d(r)=\left\{\begin{array}{cl}
\rho_{0} & \text { for } \quad r>r_{s} \\
0 & \text { for } \quad r<r_{s}
\end{array}\right.
$$

where $\rho_{0}=D\left(3 / 4 \pi r_{s}^{3}\right)$. The value of $r_{s}$ is equivalent to the radius of the Wigner-Seitz sphere. The coefficient $D$ is chosen in such a way as to obtain the correct normalization of $\psi_{2}(r)$ within the Wigner-Seitz sphere. $V_{\mathrm{HL}}(\rho)$ is the Hedin-Lundqvist exchange-correlation correction for an electron gas of density $\rho$.

\section{The effect of electron-positron interaction}

In the presence of the positron the electronic structure is described by two functions $\chi_{i}\left(r_{p}, r\right)$, where $r_{p}$ are parameters corresponding to the positron coordinates. $\chi_{i}$ obey the equations

$$
\begin{aligned}
& {\left[-\frac{1}{2} \nabla^{2}+V(r)+\frac{1}{2} W\left(r_{p}, r\right)\right] \chi_{1}\left(r_{p}, r\right)=E_{1}\left(r_{p}\right) \chi_{1}\left(r_{p}, r\right),} \\
& {\left[-\frac{1}{2} \nabla^{2}+V(r)+\frac{1}{2} W\left(r_{p}, r\right)\right] \chi_{2}\left(r_{p}, r\right)=0,}
\end{aligned}
$$

where $W\left(r_{p}, r\right)$ is the screened potential of electron-positron interaction. $\chi_{i}$ indicate the form of the functions $\psi_{i}$ in presence of the positron.

From test calculations it follows that the effect of positron interaction with conduction electrons depends only slightly on the polarization of the core by the positron. Also the state of the core depends very weakly on the state, and even on the presence, of valence electrons. This is why the equations for $\chi_{1}$ and $\chi_{2}$ can be solved separately. This allowed computing the enhancement amplitudes for conduction (Fig. 1) as well as for core electrons (Fig. 2). The polarization of the core by the positron has been found also in elements where the distribution of valence electrons can hardly be expressed by single density amplitude, provided the core contains only s-electrons. The results for different elements are shown in Fig. 2. Since core and valence electrons interact with the positron to a large 


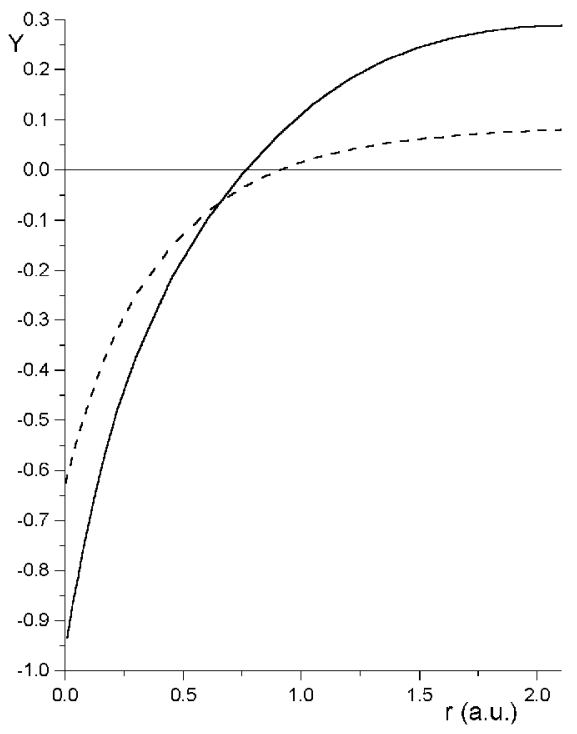

Fig. 1. The density amplitude of conduction electrons on an atom of lithium embedded in jellium $\psi_{2}(r)$ (dashed curve) and as seen by the positron $\chi_{2}(r, r)$ (full curve).
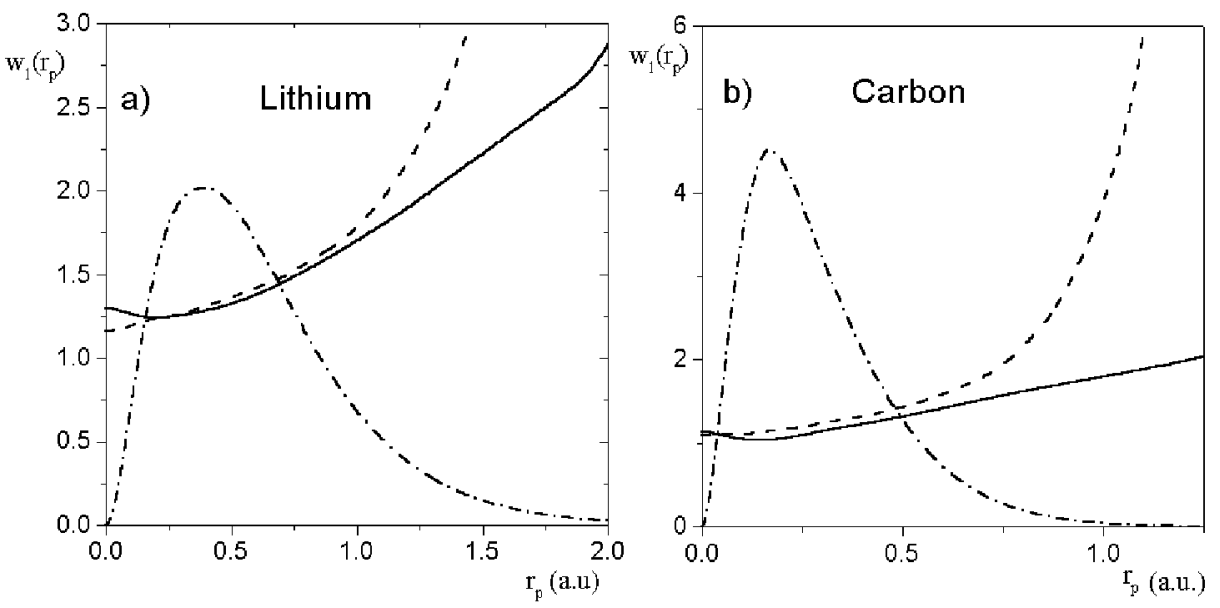

Fig. 2. The enhancement amplitude of the core electrons for different atoms embedded in jellium according to this work.

degree independently, the local density approximation could be replaced by the local partial density approximation (LPDA), where only core electrons or only valence electrons were included in the density.

As shown in Figs. 1 and 3 the enhancement amplitude is greatly affected by the displacement of the node (from 0.9 to 0.76 a.u.) due to interaction with the positron. Such a displacement of the nodes has been observed by Chiba in 


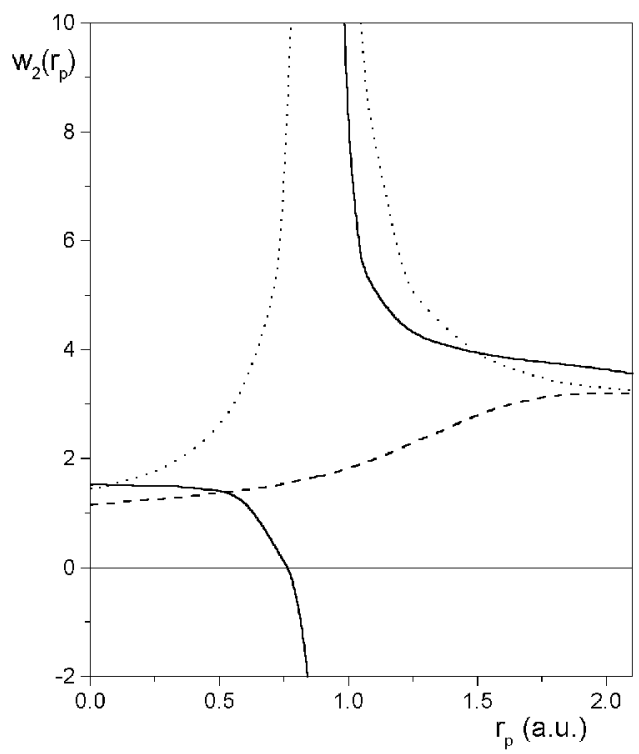

Fig. 3. The enhancement amplitude of conduction electrons in Li in our model (full curve) and according to LPDA (dotted curve). The LDA enhancement amplitude is shown by the dashed curve.

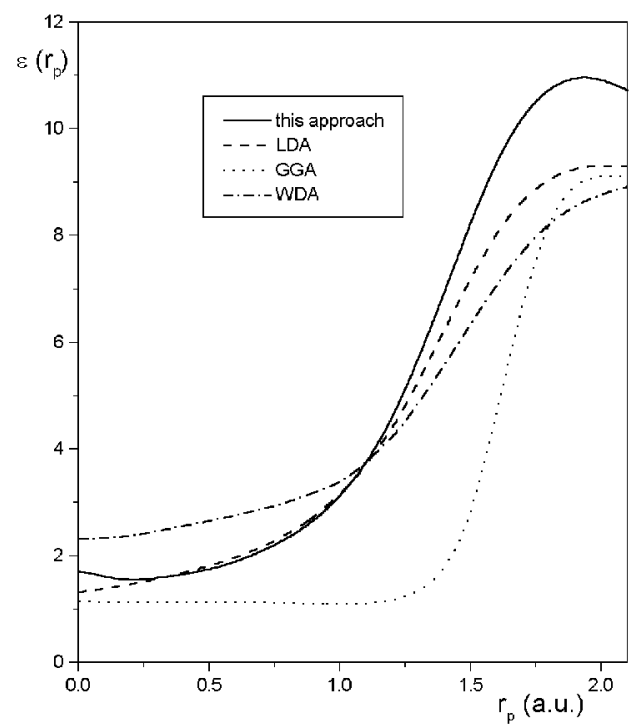

Fig. 4. Effective enhancement $\varepsilon\left(r_{p}\right)$ inside an atom of lithium embedded in an electron gas calculated in this work (solid curve) and according to LDA (dashed curve), GGA (dotted curve), and WDA (dashed-dotted curve).

$\mathrm{MgO}$ [13]. However, the effective enhancement which takes into account also the polarization of the core by the positron behaves quite regularly (Fig. 4). 


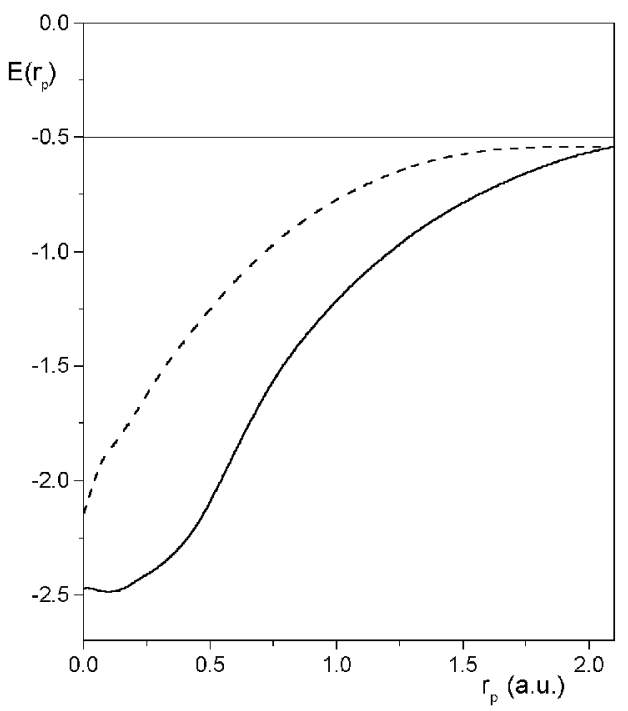

Fig. 5. Comparison of $E\left(r_{p}\right)=2 E_{1}\left(r_{p}\right)+E_{\mathrm{v}}$ (full curve) and the energy of electron-positron correlation in lithium according to LDA (dashed curve). The energies have been normalized in such a way as to coincide at $r_{p}=2.1$.

The energy of the polarized core state $E_{1}\left(r_{p}\right)$ includes the effective attraction (of chemical character) between the positron and the nucleus due to collectivization of core electrons. It will enter the positron Hamiltonian as a contribution to the positron potential. It is shown in Fig. 5 (after normalization taking into account a constant contribution $E_{\mathrm{v}}$ of conduction electrons) and compared to LDA predictions concerning $\mathrm{e}^{+}-\mathrm{e}^{-}$correlation energy.

\section{Conclusions}

It is well known that positron annihilation rates in metals are lower than expected on the ground of the local density approximation. This is why Mijnarends et al. [11] are in favor of according more confidence to the theoretical calculations of Lantto [14]. In Lantto's calculations the oversimplified trial wave function of the Jastrow type is used for describing the many electrons-one positron system; the results of Lantto form the basis for the formulas of Boroński and Nieminen [15] and of Puska et al. [16] that describe the electron-positron enhancement factors as functions of the electron density. The less controversial calculations of Arponen and Pajanne [17] and also of Lowy and Jackson [18] and Rubaszek and Stachowiak [19] lead to markedly higher annihilation rates. A discussion of this crucial problem can be found in Appendix.

The present work, however, is not devoted to studying merits of different approaches to $\mathrm{e}^{+}-\mathrm{e}^{-}$interaction in an electron gas, but to trying to understand specific properties of the behavior of a positron in a real metal in order to develop an attitude with regard to such heuristic approaches to this problem as LDA, GGA, and WDA. 
In this context the local annihilation rates shown in Fig. 4 seem to reproduce quite well the predictions of the local density approximation. However, the enhancement factors for different electron populations differ substantially from LDA predictions (Figs. 2 and 3 ). Figure 2 points at a lower enhancement factor in the tail of the core electron distribution, most important for positron annihilation. This would give in the positron lifetime an effect going in the right direction (decrease in the total annihilation rate). However, in Fig. 5 we observe a higher energy of electron positron correlation than predicted by LDA. This effect is due to collectivization of core electrons between the nucleus and the positron. It would facilitate penetration of the positron into the core region characterized by high electron density, decreasing in this way the positron lifetime.

The technical mathematical problems solved in the present work should facilitate further studies of $\mathrm{e}^{+}-\mathrm{e}^{-}$interaction in solids.

\section{Acknowledgments}

This work benefited of grant No. 2 P03B 09912 of the Committee for Scientific Research.

\section{Appendix}

\section{Comments on existing theories of $e-p$ interaction in an electron gas and their application to real metals}

Some of the results of former theoretical investigations of the e-p interaction in jellium are in permanent use in the interpretation of the experimental data. This needs, however, critical comments.

The present state of the art can be characterized best basing on the recent work of Mijnarends et al. [9] who investigated high momentum annihilation with core electrons with two-detector studies of the Doppler broadening. The interpretation based on assuming three models of application to the real metal (Al) the enhancement factors found for jellium. The first model was the LDA of the enhancement of Arponen and Pajanne (AP) [17] given by the formula [7]

$$
\gamma=1+1.23 r_{s}-0.0742 r_{s}^{2}+r_{s}^{3} / 6
$$

The second $\left(\mathrm{LDA}^{\prime}\right)$ was the same type approximation but given by the formula of Puska et al. [16]

$$
\gamma=1+1.23 r_{s}+0.9889 r_{s}^{3 / 2}-1.482 r_{s}^{2}+0.3956 r_{s}^{5 / 2}+r_{s}^{3} / 6
$$

which numerically differed very little from the well-known formula of Boroniski and Nieminen [15]

$$
\gamma=1+1.23 r_{s}+0.8295 r_{s}^{3 / 2}-1.26 r_{s}^{2}+0.3286 r_{s}^{5 / 2}+r_{s}^{3} / 6
$$

The third model was the gradient correction (GGA) (containing an experimentally determined parameter) to the AP factors, introduced by Barbiellini et al. [7]. 
Mijnarends et al. [9] found that the $\mathrm{LDA}^{\prime}$ model gives the best agreement with experiment, though a small correction by applying a lesser than in GGA gradient correction could probably lead to some improvement.

What do the results of Ref. [9] mean?

The complete theory of $\mathrm{e}-\mathrm{p}$ interaction should be self-consistent, it should take into account the momentum dependence of electron scattering and the effect of the $\mathrm{e}-\mathrm{e}$ correlation on the $\mathrm{e}-\mathrm{p}$ interaction. The three necessary features (TNF) enumerated above form the basis of the calculations of Lowy and Jackson [18] using the Lippman-Schwinger equation and of the work of Rubaszek and Stachowiak [19] which includes self-consistency and $\mathrm{e}^{-}-\mathrm{e}^{-}$correlations into the Bethe-Goldstone formalism of Kahana [20]. Both works in spite of using approaches quite different from each other and from AP end with almost the same enhancement factors described by formula (A1) which is also the result of TNF calculation.

Some groups of physicists do not like the approach of Kahana to EPI, because it assumes a rectangular electron distribution in momentum space; they prefer a Daniel-Vosko type distribution. Our feeling is that it is not obvious that the electron holes below and the electron states above the Fermi surface which both are due to electron-electron correlations are enhanced to the same degree as the main parts of the electron wave functions with well defined momenta which describe the rectangular distribution. Both theoretical considerations of Carbotte and Kahana [21, 22] and experimental studies (e.g. [23, 24]) support the above point of view, in contrast to the Compton scattering in which the nonrectangular Daniel-Vosko distribution was detected relatively easily [25]. This does not mean that deviation from rectangular shape cannot be observed by positron annihilation [26]. One can expect that for higher densities the Daniel-Vosko distribution will be less affected by EPI. Our opinion basing on the calculations of Boroński [27] (cf. also [24]) is that the Daniel-Vosko effect in positron annihilation in an electron gas is poorly treated both in works using a rectangular occupation number for electrons $[9,8,7,28]$ where it is neglected and by Arponen and Pajanne which disturb with the positron the Sawada boson vacuum corresponding to the random phase approximation (RPA) state. As follows from the calculations of Boroniski there is no evidence that they are able to include in their computations the influence of $\mathrm{e}^{+} \mathrm{e}^{-}$ interaction on the Daniel-Vosko distribution (as seen by positrons). They just reproduce the RPA results. This could be done as well using the Bethe-Goldstone formalism. Only Monte Carlo calculations could, in principle, give an answer.

Since three independent TNF calculations, using very different approaches to EPI led to quite similar enhancement factors, one must conclude that the formula (A1) is an unavoidable consequence of the three requirements.

Let us observe that Lantto's calculations [14] basing faithfully on a Jastrow type trial function include certainly one from the TNF assumptions, namely self-consistency. In the case of a system of one positron and $N$ electrons the trial function (1) of Lantto has the form

$$
\Psi=F_{12} F_{11} \phi_{1}
$$

where the factors $F_{11}$ and $F_{12}$ are expressed by the function-parameters $f_{11}$ and 
$f_{12}$ through the formulae

$$
F_{11}=\prod_{i j} f_{11}\left(\boldsymbol{r}_{i}, \boldsymbol{r}_{j}\right), \quad F_{12}=\prod_{i} f_{12}\left(\boldsymbol{r}_{i}, \boldsymbol{r}_{p}\right) .
$$

$\phi_{1}$ is the Slater determinant of free electrons, $\boldsymbol{r}_{i}$ and $\boldsymbol{r}_{p}$ are the coordinates of the electrons and the positron, respectively. It is obvious that the variational function-parameter $f_{11}$ offers no possibility to minimize the Hamiltonian with regard to the dependence of $\mathrm{e}^{+}-\mathrm{e}^{-}$correlations on the distance from the positron. Also the function-parameter $f_{12}$ does not include the momentum dependence of electron scattering on the positron.

The conclusion from the results of Ref. [9] (and many previous measurements - since the formula of Boroński and Nieminen is so popular among positron physicists) is that some effects not included in TNF lead to lowering the annihilation rate of positrons in metals (and also in an electron gas). Let us remark, however, that the results of Lantto differ from those of AP no more than by $20 \%$, while Lantto himself admits that his correlation functions are accurate up to about $10 \%$, besides his published calculations are performed for $r_{s} \leq 5$, leaving aside the electron densities of cesium and rubidium. Our feeling is that in order to check the reliability of the results, calculations should be performed up to $r_{s}=8$ or so.

Whenever in our calculations we neglected electron-electron correlations, or rather their dependence on the positron position this resulted in lowering the annihilation rate (cf. [10] and unpublished calculations). Indeed, subtracting from

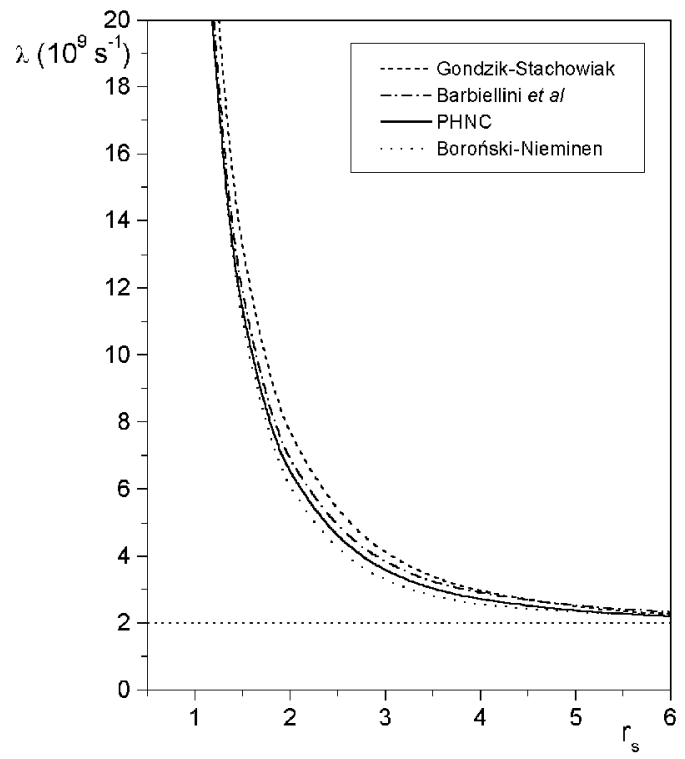

Fig. 6. Comparison of different formulas for the positron annihilation rate $\lambda\left(r_{s}\right)$ in an electron gas (dashed curve - Gondzik-Stachowiak [10], dashed-dotted curve - Barbiellini et al. [7], solid curve - PHNC [28], dotted curve - Boroński-Nieminen [15]). 
the screening cloud around the positron a part of it due to the correlation hole increases the contact density of electrons on the positron owing to lesser screening of its positive charge.

Thus, if one compares e.g. the curves labelled Gondzik-Stachowiak and of formula (A1) in Fig. 6 one concludes that allowing for momentum dependence of electron scattering on the positron leads to lowering the annihilation rate. Therefore from this point of view the agreement of Lantto's results with experiment is not physically enlightening and can be treated as purely accidental and following from compensation of different effects that have been neglected. This limits essentially the physical impact of the statement of Mijnarends et al. [9] that "... only the local density enhancement of Puska, Seitsonen, and Nieminen can simultaneously reproduce the profile and the lifetime...". Let us remark, however, that the form (A4) of the trial function does not exclude the $r$ dependence of $\mathrm{e}^{+}-\mathrm{e}^{-}$correlations (where $r$ is the distance from the positron). Indeed, the Hartree-Fock approach to electronic structure neglects $\mathrm{e}^{-}-\mathrm{e}^{-}$correlation factors altogether, obtaining nevertheless the exchange correction to the effective potential. On the other hand, the Kohn-Sham approach uses (in local density approximation) solutions of the many electron problem for all densities of the electron gas occurring in the system. The Jastrow trial function in the case of one positron in the electron gas approach is able to utilize the solution of the $\mathrm{e}^{-}-\mathrm{e}^{-}$correlation problem only for the average density of the electron gas.

We advocate for the another TNF calculations presented in Ref. [28], which also yielded enhancement factors. The wave function of the system consisted of the Slater determinant built from one-electron wave functions $\phi$ modulated by enhancement amplitudes $w$

$$
\Psi\left(s_{1}, \ldots s_{N}\right)=\prod_{i=1}^{N} w\left(s_{i}\right)\left|\begin{array}{cccc}
\phi_{1}\left(s_{1}\right) & \phi_{1}\left(s_{2}\right) & \ldots & \phi_{1}\left(s_{N}\right) \\
\phi_{2}\left(s_{1}\right) & \phi_{2}\left(s_{2}\right) & \ldots & \phi_{2}\left(s_{N}\right) \\
\ldots & \ldots & \ldots & \ldots \\
\phi_{N}\left(s_{1}\right) & \phi_{N}\left(s_{2}\right) & \ldots & \phi_{N}\left(s_{N}\right)
\end{array}\right|
$$

and

$$
\boldsymbol{s}_{i}=\boldsymbol{r}_{i}-\boldsymbol{r}_{p} .
$$

The one-electron wave functions are allowed to be weakly nonorthogonal. Therefore the electron density is computed from the formula

$$
\rho(s)=2 \sum_{\boldsymbol{k}_{\mathrm{occ}}} \Psi_{\boldsymbol{k}}^{*}(s) \Psi_{\mathbb{k}}(s)-2 \sum_{\boldsymbol{k}^{\prime} \neq \mathbb{k}_{\mathrm{occ}}} A_{\mathbb{k}^{\prime} \boldsymbol{k}} \Psi_{\boldsymbol{k}}^{*}(s) \Psi_{\mathbb{k}}(s),
$$

where

$$
\begin{aligned}
& A_{\boldsymbol{k}^{\prime} \boldsymbol{k}}=\int \mathrm{d} s^{\prime} \psi_{\boldsymbol{k}}^{*}\left(s^{\prime}\right) \psi_{\boldsymbol{k}^{\prime}}\left(s^{\prime}\right), \\
& \psi_{\boldsymbol{k}^{\prime}}(s)=w(s) \phi_{\boldsymbol{k}_{\boldsymbol{k}}}(s), \\
& \phi_{\boldsymbol{k}}(s)=\frac{1}{\sqrt{\Omega}} \mathrm{e}^{\mathrm{i} k s}+\nu_{\boldsymbol{k}}(s) .
\end{aligned}
$$


The corresponding enhancement factors can be described by the formula

$$
\gamma=1+1.23 r_{s}-0.1375 r_{s}^{2}+r_{s}^{3} / 6
$$

The behavior of different enhancement factors is shown in Fig. 6.

Thus Ref. [28] presents the only TNF enhancement factors that are appreciably lower than the ones of formula (A1). Therefore, as concerns an electron gas, the results of Ref. [9] point at the importance of allowing for weak nonorthogonality of one-electron wave functions, which results in their interaction with the positron. This problem was first called attention upon by Lowy and Jackson [18]. Let us remark that this approach (referred to as perturbed hypernetted-chain (PHNC)) does not introduce artificially the Pauli exclusion principle unlike the approach of Kahana [21] criticized for this reason in Ref. [18].

As concerns deviations from the local density approximation in metal lattices, this problem according to Ref. [9] is of lesser importance (we have also examined the GGA performing some model calculations). But this conclusion follows from experimental considerations and means that a satisfactory theory of $\mathrm{e}^{+}-\mathrm{e}^{-}$interaction in metal lattices is not existing.

\section{References}

[1] J.P. Carbotte, Phys. Rev. 144, 309 (1966).

[2] H. Sormann, Phys. Rev. B 54, 4558 (1996).

[3] J. Arponen, P. Hautojärvi, R. Nieminen, E. Pajanne, J. Phys. F 3, 2092 (1973).

[4] E. Bonderup, J.U. Andersen, D.N. Lowy, Phys. Rev. B 20, 883 (1979).

[5] S. Daniuk, G. Kontrym-Sznajd, J. Mayers, A. Rubaszek, H. Stachowiak, P.A. Walters, R.N. West, J. Phys. F 17, 1365 (1987).

[6] K.O. Jensen, J. Phys., Condens. Matter 1, 10595 (1989); S. Daniuk, M. Sob, A. Rubaszek, Phys. Rev. B 43, 2580 (1991); B. Barbiellini, P. Genoud, T. Jarlborg, J. Phys., Condens. Matter 3, 7631 (1991); M.J. Puska, J. Phys., Condens. Matter 3, 3455 (1991).

[7] B. Barbiellini, M.J. Puska, T. Torsti, R.M. Nieminen, Phys. Rev. B 51, 7341 (1995); B. Barbiellini, M.J. Puska, T. Korhonen, A. Harju, T. Torsti, R.M. Nieminen, Phys. Rev. B 53, 16201 (1996).

[8] A. Rubaszek, Z. Szotek, W.M. Temmerman, Phys. Rev. B 58, 11285 (1998).

[9] P.E. Mijnarends, A.C. Kruseman, A. van Veen, H. Schut, A. Bansil, J. Phys., Condens. Matter 10, 10383 (1998).

[10] J. Gondzik, H. Stachowiak, J. Phys. C 18, 5399 (1985).

[11] A. Kallio, P. Pietiläinen, L. Lantto, Phys. Scr. 25, 943 (1982); P. Pietiläinen, A. Kallio, Phys. Rev. B 27, 224 (1983).

[12] H. Stachowiak, E. Boroński, G. Banach, Mater. Sci. Forum 255-257, 408 (1997); H. Stachowiak, E. Boroński, G. Banach, Phys. Rev. B 62, 4431 (2000).

[13] T. Chiba, Proc. 12 Int. Conf. on Positron Annihilation (ICPA 12), München 2000, to be published in Mater. Sci. Forum.

[14] L. Lantto, Phys. Rev. B 36, 5160 (1987).

[15] E. Boroński, R.M. Nieminen, Phys. Rev. B 34, 3820 (1986). 
[16] M.J. Puska, A.P. Seitsonen, R.M. Nieminen, Phys. Rev. B 52, 10947 (1995).

[17] J. Arponen, E. Pajanne, Ann. Phys. (New York) 121, 343 (1979).

[18] D.N. Lowy, A.D. Jackson, Phys. Rev. B 12, 1689 (1975).

[19] A. Rubaszek, H. Stachowiak, Phys. Rev. B 38, 3846 (1988).

[20] S. Kahana, Phys. Rev. 129, 1622 (1963).

[21] J.P. Carbotte, S. Kahana, Phys. Rev. 139, A213 (1965).

[22] J.P. Carbotte, in: Positron Solid State Physics, Eds. W. Brandt, A. Dupasquier, North-Holland, Amsterdam 1983, p. 32.

[23] T. Hyodo, T. McMullen, A.T. Stewart, Phys. Rev. B 33, 3050 (1986).

[24] H. Stachowiak, A. Rubaszek, in: Positrons at Metallic Surfaces, Ed. A. Ishii, series Solid State Phenomena, Vol. 28/29, Trans. Tech. Publ., Aedermannsdorf 1993, p. 7 .

[25] N. Shiotani, Mater. Sci. Forum 175-178, 839 (1995).

[26] A.A. Manuel, D. Vasumathi, B. Barbiellini, A. Shukla, P. Suortti, T. Chiba, Mater. Sci. Forum 255-257, 760 (1997).

[27] E. Boroński, Mater. Sci. Forum 105-110, 181 (1992).

[28] H. Stachowiak, Phys. Rev. B 41, 12522 (1990); H. Stachowiak, J. Lach, Phys. Rev. B 48, 9828 (1993); E. Boroński, H. Stachowiak, Phys. Rev. B 57, 6215 (1998). 